\title{
Microdysgenesis in surgical specimens from patients with epilepsy: occurrence and clinical correlations
}

\author{
Claes Nordborg, Sofia Eriksson, Bertil Rydenhag, Paul Uvebrant, Kristina Malmgren
}

Institute of Laboratory Medicine, Department of Pathology C Nordborg

Institute of Clinical Neuroscience, Department of Neurology S Eriksson

K Malmgren

Department of Neurosurgery, Institute of Selected Clinical Sciences B Rydenhag

Department of Pediatrics, Sahlgrenska University Hospital, Göteborg, Sweden

P Uvebrant

Correspondence to: Dr Claes Nordborg, Institute of Laboratory Medicine, Department of Pathology, Sahlgrenska University Hospital, S-413 45 Göteborg, Sweden. Telephone 00463134266 14; fax 0046314172 83; email

claes.nordborg@ss.gu.se

Received 27 November 1997 and in revised form 16 March 1999 Accepted 14 April 1999

\begin{abstract}
Malformations of cortical development are commonly associated with epilepsy. In the first 139 consecutive patients in the Göteborg epilepsy surgery series, parenchymal malformations were found in $56.1 \%$ of the children and in $23.1 \%$ of the adults. Microdysgenesis (MDG), which was the most common parenchymal malformation, was found in $35.1 \%$ of the children and in $16.7 \%$ of the adults. The aim of this study was to identify clinical characteristics of patients with MDG. Mental retardation was found to be significantly more common in patients with major parenchymal malformations and in patients with MDG compared with patients without parenchymal malformations. $\mathbf{P a}-$ tients with major parenchymal malformations as well as patients with MDG also had a significantly earlier onset of seizures than patients without parenchymal malformations, also when adjusting for mental retardation. Patients with MDG were in these clinical aspects shown to closely resemble patients with major malformations. These findings suggest that MDG is a pathoanatomical entity of clinical relevance, with implications both in mental retardation and in epileptogenesis. (F Neurol Neurosurg Psychiatry 1999;67:521-524)
\end{abstract}

Keywords: epilepsy; microdysgenisis; mental retardation

Malformations of the cerebral cortex are being recognised with increased frequency as a cause of epilepsy and also of mental retardation and other neurodevelopmental disorders. ${ }^{1}$ As neuroimaging techniques develop, more subtle cortical malformations are demonstrated in patients with pharmacoresistant epilepsy. ${ }^{12}$ However, there is still a proportion of patients in whom no structural correlates can be found despite high quality MRI. ${ }^{2-4}$ In these cases further histopathological analyses using modern techniques are of special importance to increase our knowledge of the aetiology of surgically treated epilepsy.

Meencke and Janz first described microdysgenesis (MDG) as a minor parenchymal malformation in patients with generalised epilepsy. ${ }^{5-7}$ It has also been found in epilepsy surgery specimens from patients with partial epilepsy. ${ }^{2-10}$ Nordborg et al described MDG in specimens from $42.4 \%$ of 33 patients operated on because of intractable partial epilepsy. ${ }^{9}$ Although the major parenchymal malformations are usually classified according to distinct macroscopical patterns ${ }^{11}{ }^{12}$ the minor malformations are classified according to specific microscopical features..$^{5-10}$

In an analysis of the clinical, radiological, and histopathological findings in the first consecutive 139 patients in the Göteborg epilepsy surgery series ${ }^{13}$ we found a high proportion of MDG. The series comprises children and adults (ranging in age from 0.9 to 62 years at the time of operation) subjected to a wide range of resective procedures. As there has long been a discussion about the pathophysiological relevance of the discrete histopathological findings of MDG, ${ }^{5-71415}$ the purpose of the present study was to identify clinical characteristics of patients with this histopathological diagnosis. Our hypothesis was that if MDG, as well as major parenchymal malformations, is of pathophysiological importance in epilepsy, it might be possible to identify clinicopathological relations.

\section{Patients and methods}

From 1987 to 1995139 patients (60 children $\leqslant 18$ years old and 79 adults) underwent surgery for epilepsy. Most of the adults had pharmacoresistant epilepsy of temporal lobe origin, without other neurological handicaps. Many of the children, however, had severe epilepsy as part of a multihandicap. In the adult population temporal lobe resections dominated $(75.9 \%)$ followed by frontal lobe resections $(15.2 \%)$. Among the children temporal lobe resections were less dominating but still the most common operation $(25.0 \%)$, followed by frontal lobe and multilobar resections (21.7\% each), hemispherectomies (13.3\%), and callosotomies (11.6\%).

In connection with the analysis of the surgical series, all specimens were re-evaluated by the first author, who was blinded regarding clinical data. Specimens from 57 children and 78 adults contained enough tissue for 
Table 1 Neuropathological diagnoses

\begin{tabular}{llll}
\hline Neuropathological diagnoses & $\begin{array}{l}\text { Total } \\
(n=236)\end{array}$ & $\begin{array}{l}\text { Children } \\
(n=108)\end{array}$ & $\begin{array}{l}\text { Adults } \\
(n=128)\end{array}$ \\
\hline Parenchymal malformations & 50 & 32 & 18 \\
$\quad$ Major malformations & 17 & 11 & 5 \\
$\quad$ Gross malformations & 10 & 7 & 2 \\
$\quad$ Focal cortical dysplasia & 4 & 2 & 2 \\
$\quad$ Tuberous sclerosis & 2 & 2 & 0 \\
$\quad$ Other & 1 & 0 & 1 \\
$\quad$ Microdysgenesis & 33 & 20 & 13 \\
Vascular malformations & 13 & 2 & 11 \\
Low grade tumours & 22 & 9 & 13 \\
Cysts & 1 & 0 & 1 \\
Malignant tumours & 1 & 0 & 1 \\
Infections & 1 & 1 & 0 \\
Hippocampal sclerosis & 24 & 6 & 18 \\
Atrophic-gliotic lesions & 124 & 58 & 66 \\
\hline
\end{tabular}

histopathological analysis. A detailed methodological description of the histopathological analysis is given elsewhere. ${ }^{13}$ Eight main categories of lesions were recognised: parenchymal malformations, vascular malformations, low grade tumours, cysts, malignant tumours, infections, hippocampal sclerosis, and other atrophic-gliotic lesions. In total there were 55 different histopathological subgroups.

Major malformations included gross parenchymal malformations (all lesions displaying a disturbance of convolutional pattern or heterotopic islands of grey matter as well as schizencephalic defects), focal cortical dysplasia, and tuberous sclerosis. Focal cortical dysplasia was defined according to Taylor et al. ${ }^{16}$ In specimens considered as microdysgenetic five different morphological aberrations were found alone or in combination: supernumerary nerve cells in layer I, ectopic nerve cells in the white matter, indistinct cortical lamination, increased neuronal columnation, and increased clustering of nerve cells in the cortex. Balloon cells were, besides other MDG aberrations, found in only one case, which was, however, free from neuronal cytomegaly. There were no cases with marginal glioneuronal heterotopia and no cases displaying a loss of demarcation between layers I and II in the present material. ${ }^{10}$ As the border between what is considered as normal brain tissue and mild microdysgenesis is not quantitatively defined, there will, in a screening situation, by necessity be cases in which the changes are too subtle or the material too limited to allow an unequivocal diagnosis of MDG. In the present series there were 22 such patients (eight children and 14 adults) in which MDG was merely suspected. These patients have not been further considered in the statistical analyses.

Significance testing of correlations was performed with Pitman's non-parametric permutation test. ${ }^{17}$ Mantel's technique of pooling ${ }^{18}$ applied to Pitman's non-parametric permutation test was used as a non-parametric partial analysis to study the correlation between two variables adjusted for a third variable. For comparisons between groups, the Mann-Whitney $U$ test and the Kruskal-Wallis test were used.

\section{Results}

Specimens were achieved from 57 children and 78 adults. The main histopathological diag- noses are listed in table 1 (subgroups for the main diagnoses other than parenchymal malformations are detailed in table $3 \mathrm{in}^{13}$ ). Most of the specimens from individual patients had more than one histopathological diagnosis. The mean number of diagnoses in the adults was 1.7 (range 1-3) and in the children 1.9 (range 1-3). The categories of lesions were the following: parenchymal malformations in $37.0 \%(\mathrm{n}=50)$, vascular malformations in $9.6 \%(n=13)$, low grade tumours in $16.3 \%$ $(n=22)$, one cyst, one malignant tumour, one infection, hippocampal sclerosis in $17.7 \%$ $(n=24)$, and other atrophic-gliotic lesions in $78.5 \%(\mathrm{n}=106)$.

Parenchymal malformations were found in $56.1 \%(n=32)$ of the children and in $23.1 \%$ $(n=18)$ of the adults. Microdysgenesis was the most common parenchymal malformation in both groups and was found in $35.1 \%(n=20)$ of the children and $16.7 \%(n=13)$ of the adults. Histopathological characteristics of MDG are illustrated in the figure.

\section{CLINICAL DATA IN RELATION TO}

\section{HISTOPATHOLOGICAL DIAGNOSES}

Mental retardation was present in $28.1 \%$ of the patients $(5.0 \%$ of the adults, $58.4 \%$ of the children). The proportion of patients with in the main histopathological groups was as follows: $48 \%(n=24)$ of the patients with parenchymal malformations were mentally retarded $(52.9 \%$ of the 17 patients with major malformations and $45.5 \%$ of the 33 patients with MDG), $4.5 \%(n=1)$ of the patients with low grade tumours, and $20.0 \%(n=5)$ of the patients with atrophic-gliotic lesion had mental retardation. There were no patients with only hippocampal sclerosis and mental retardation. The proportion of mental retardation was significantly higher among the patients with parenchymal malformations $(p<0.0001)$ than among those without, and this was the case both for major malformations $(\mathrm{p}<0.02)$ and MDG $(\mathrm{p}<0.01)$.

Early seizure onset was significantly related to the presence and the severity of mental retardation $(\mathrm{p}<0.0001)$. Age at seizure onset also varied between the main histopathological groups as detailed in table 2. Patients with parenchymal malformations (major malformations and MDG) had a significantly earlier seizure onset than patients without parenchymal malformations $(p<0.0001)$, and this held true also when subdividing into major malformations $(\mathrm{p}<0.001)$ and MDG $(\mathrm{p}<0.001)$.

When adjusting for mental retardation, the patients with parenchymal malformations (major and MDG) still had a significantly earlier seizure onset $(p<0.001)$ than the patients without parenchymal malformations. When subdividing into major parenchymal malformations and MDG, the seizure onset was still significantly earlier $(\mathrm{p}<0.05 v \mathrm{p}<0.001)$ than for the patients without parenchymal malformations.

\section{Discussion}

The pathogenetic relevance of microdysgenesis in epilepsy has been a matter of controversy for many years. ${ }^{5-81415}$ It has been argued that 

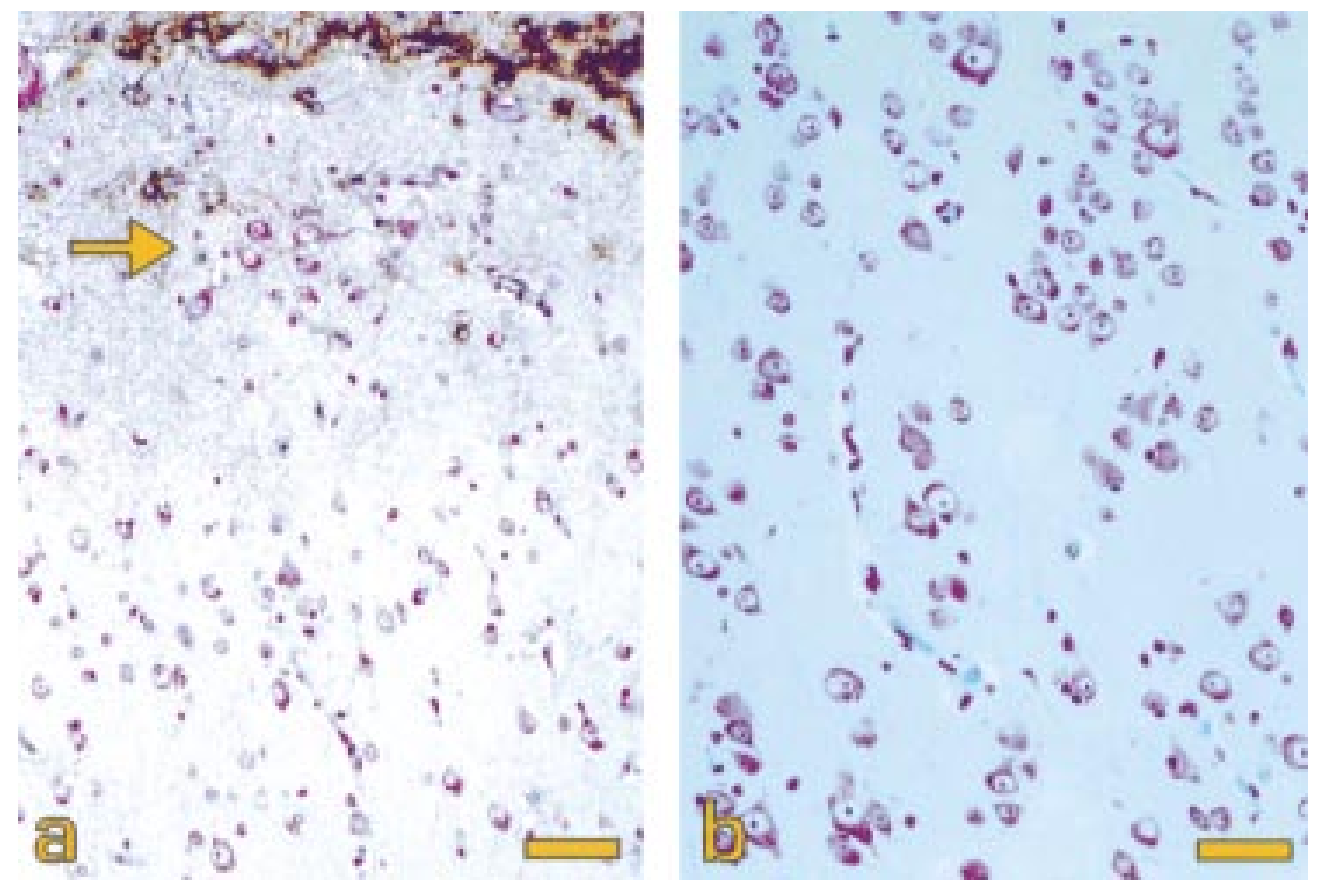

(A) Specimen from the right frontal lobe of a 36 year old man with severe pharmacoresistant epilepsy since the age of six and borderline intellectual level. A group of supernumerary nerve cells is indicated by an arrow in layer I (top half). Note brown stained subpial glia (top). (Anti-GFAP and cresyl violet. Bar=65 $\mu \mathrm{m}$.) (B) Specimen from the left temporal lobe of a 5 year old boy with severe pharmacoresistant epilepsy since birth and mild mental retardation. Irregular, clustered cortical nerve cell distribution and random neuronal orientation. Cortical surface upwards. (Luxol fast blue-cresyl violet. Bar=46 $\mu \mathrm{m}$.)

discrete aberrations in the cortical architecture can be seen also in subjects without epilepsy and might represent one end of the normal variation. According to published postmortem control series, MDG is, however, more common in epilepsy material. In one postmortem study, MDG was found in $37.7 \%$ of 591 patients with epilepsy and in only $6 \%$ of 150 controls. ${ }^{19}$ In another report severe neuronal ectopia (defined as more than eight dystopic neurons $/ 2 \mathrm{~mm}^{2}$ ) was found in $42 \%$ of specimens from the temporal lobes of 50 surgically treated patients whereas there were no microdysgenetic changes in 33 postmortem controls. ${ }^{8}$

There are some independent data about the structure of microdysgenetic cortex in patients with epilepsy that can have a bearing on the pathogenetic relevance of MDG. In an earlier detailed study of specimens from 11 patients with epilepsy in our series, using confocal laser scanning microscopy, an increased number of dendritic abnormalities of single pyramidal and non-pyramidal cells could be demonstrated in the seven patients with MDG but not in those without $M D G{ }^{20}$ This finding illustrates that MDG is not only a question of aberrant nerve cell distribution, but that individual neurons and neuronal connections may be abnormal. It

Table 2 Age (years) at seizure onset in relation to histopathological diagnosis

\begin{tabular}{lll}
\hline & Median & Range $\left(P_{25}-P_{75}\right)$ \\
\hline Parenchymal malformations & 3.0 & $(0.5-7.0)$ \\
$\quad$ Major malformations & 0.7 & $(0.3-7.0)$ \\
$\quad$ Microdysgenesis & 3.5 & $(0.8-6.8)$ \\
Vascular malformations & 24.5 & $(17.5-36.0)$ \\
Low grade tumours & 9.3 & $(4.3-18.8)$ \\
Hippocampal sclerosis & 22.5 & $(6.5-26.5)$ \\
Atrophic-gliotic lesions & 12.0 & $(4.0-17.0)$ \\
\hline
\end{tabular}

$\mathrm{P}_{25}=25$ th percentile; $\mathrm{P}_{75}=75$ th percentile. seems reasonable to assume that such structural abnormalities and misconnections could lead to abnormal function.

Another way to test the possible pathogenetic relevance of MDG was used in the present investigation in which the relation between MDG and clinical variables was studied. In earlier studies patients with more severe malformations of cortical development, changes have been shown to have a higher incidence of mental retardation or developmental delay and a higher seizure frequency than those with less severe histopathological changes. ${ }^{2}{ }^{410}{ }^{12}$ In the one earlier study in which clinical correlates to MDG were studied, age at onset was not shown to relate to a histopathological diagnosis of MDG. ${ }^{8}$

In the present study patients with MDG were shown to closely resemble patients with major malformations in several clinical aspects. They were significantly more often mentally retarded, and had a significantly lower age at seizure onset than the patients without parenchymal malformations. The age at seizure onset was still significantly lower for patients with MDG when adjusting for mental retardation and can hence not be attributed to some unknown underlying cause for mental retardation other than MDG. These findings support the contention that MDG is a pathoanatomical entity of clinical relevance, probably implied both in mental retardation and in epileptogenesis.

Because the difference in nerve cell distribution between normal and microdysgenetic brain is a matter of degree rather than quality, light microscopic screening of epileptic brain tissue will not enable an absolute delineation between microdysgenetic and normal brain. To 
avoid overinterpretation, we only included the 33 unequivocal cases in the statistical analyses. However, MDG might still be underdiagnosed in our material, as indicated by the 22 cases considered to have "suspected MDG". Furthermore, in the present study the microdysgenetic changes have not been graded or quantified. It may well be that the clinicopathological correlations of MDG are related to the severity of the microdysgenetic changes. Thus a more detailed quantitative assessment of the nerve cell distribution in epileptic brain tissue is necessary for a finer analysis of the relationship between mild neuronal migration defects and various clinical variables.

We express our gratitude to the following people: Lisbeth Gustafsson, Fariba Moraghebi, and Margareta Persson for excellen technical assistance; Nils-Gunnar Pehrsson for statistical expertise; Olof Ostensson for data analysis; and Lisbeth Lindberg for assistance with preparation of the manuscript. The study was supported by the Regional Health Care Authority of West Sweden and the First of Mayflower Annual Campaign.

1 Kuzniecky RI. Magnetic resonance imaging in developmen-

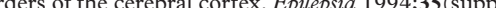
6):S44-56.

2 Raymond AA, Fish DR, Sisodiya SM, et al. Abnormalities of gyration, heterotopias, tuberous sclerosis, focal cortical dysplasia, microdysgenesis, dysembryoplastic neuroepithelial tumour and dysgenesis of the archicortex in epilepsy. Clinical, EEG and neuroimaging features in 100 adult Clinical, EEG and neuroimaging

3 Meencke HJ. Minimal developmental disturbances in epilepsy and MRI. In: Shorvon SD, Fish DR, Andermann $\mathrm{F}$, et al, eds. Magnetic resonance scanning and epilepsy. New York: Plenum Press, 1994:127-36.

4 Wyllie E, Baumgartner C, Prayson R, et al. The clinical spectrum of focal cortical dysplasia and epilepsy. $\mathcal{F}$ Epilepsy 1994;7:303-12. 5 Meencke HJ, Janz D. Neuropathological findings in primary
generalized epilepsy: a study of eight cases. Epilepsia 1984; 25:8-21.
6 Meencke HJ. Neuron density in the molecular layer of the frontal cortex in primary generalized epilepsy. Epilepsia 1985;26:450-4

7 Meencke HJ. The density of dystopic neurons in the white matter of the gyrus frontalis inferior in epilepsies. F Neurol 1983;230:171-81.

8 Hardiman O, Burke T, Phillips J, et al. Microdysgenesis in resected temporal neocortex: incidence and clinical significance in focal epilepsy. Neurology 1988;38:1041-7.

9 Nordborg C, Sourander P, Silfvenius H, et al. Mild cortical dysplasia in patients with intractable partial seizures: a histological study. In: Wolf P, Dam M, Janz D, Dreifuss FE, eds. Advances in epileptology. Vol 16. New York: Raven Press, 1987:29-33.

10 Mischel PS, Nguyen LP, Vinters HV. Cerebral cortical dysplasia associated with pediatric epilepsy. Review of neuropathologic features and proposal for a grading system. F Neuropathol Exp Neurol 1995;54:137-53.

11 Barth PG. Disorders of neuronal migration. Can $\mathcal{F}$ Neurol Sci 1987; 14:1-16.

12 Barkovich AJ, Kuzniecky RI, Dobyns WB, et al. A classification scheme for malformations of cortical developclassification scheme for malformat

13 Eriksson S, Malmgren K, Rydenhag B, et al. Surgical treatment of epilepsy: clinical, radiological and histopathological findings in 139 children and adults. Acta Neurol Scand 1999;99:8-15.

14 Lyon G, Gastaut H. Considerations on the significance attributed to unusual cerebral histological findings recently described in eight patients with primary generalized epilepsy. Epilepsia 1985;26:365-7.

15 Meencke HJ, Janz D. The significance of microdysgenesia in primary generalized epilepsy: an answer to the considerations of Lyon and Gastaut. Epilepsia 1985;26(4):368-371.

16 Taylor DC, Falconer MA, Bruton CJ, et al. Focal dysplasia of the cerebral cortex in epilepsy. F Neurol Neurosurg Psychiatry 1971;34:369-87.

17 Bradley JV. Distribution-free statistical tests. London: PrenticeHall, 1968:68-86.

18 Mantel N. Chi-square test with one degree of freedom. Fournal of the American Statistical Association 1963:690700 .

19 Meencke H-J, Veith G. Migration disturbances in epilepsy. In: Engel Jr J, Wasterlain C, Cavalheiro EA, et al, eds.
Molecular neurobiology of epilepsy. Elsevier, 1992:31-40.

20 Belichenko PV, Sourander P, Malmgren K, et al. Dendritic morphology in epileptogenic cortex from TRPE patients, revealed by intracellular lucifer yellow microinjection and confocal laser scanning microscopy. Epilepsy Res 1994;18: 233-47. 